\title{
Delay characteristics of surface charging on a cylindrical insulator in vacuum
}

\section{$\operatorname{AUTHOR}(\mathrm{S})$ :}

Yamamoto, O; Takuma, T; Kakehashi, Y; Ikoma, S; Shioiri, T

\section{CITATION:}

Yamamoto, $O$... [et al]. Delay characteristics of surface charging on a cylindrical insulator in vacuum. IEEE TRANSACTIONS ON DIELECTRICS AND ELECTRICAL INSULATION 2000, 7(6): 812-817

\section{ISSUE DATE:}

2000-12

URL:

http://hdl.handle.net/2433/39975

\section{RIGHT:}

(c)2000 IEEE. Personal use of this material is permitted. However, permission to reprint/republish this material for advertising or promotional purposes or for creating new collective works for resale or redistribution to servers or lists, or to reuse any copyrighted component of this work in other works must be obtained from the IEEE. 


\title{
Delay Characteristics of Surface Charging on a Cylindrical Insulator in Vacuum
}

\author{
O. Yamamoto, T. Takuma, Y. Kakehashi, S. Ikoma \\ Kyoto University \\ Kyoto, Japan
}

and T. Shioiri

Toshiba Co.

\begin{abstract}
This paper describes the temporal characteristics of surface charging on a cylindrical insulator made of polymethylmethacrylate (PMMA) resin or alumina ceramic. The insulator is subjected to a dc voltage step or to a trapezoidal voltage in vacuum. The charging is observed by using an electrostatic probe located at the surface of the cathode close to the triple junction where the insulator, cathode and vacuum meet. The probe signal shows a step increase associated with the charging. The interval between the voltage rise and the charging, which is defined as the delay time of charging in this paper, ranges from $10^{-5} \mathrm{~s}$ to $10 \mathrm{~s}$ depending on the material and the applied voltage. It also depends on the condition of the insulator-cathode interface, the electrical prestress and the surface roughness of the insulator. Among these factors, the surface roughness has the largest effect on the delay time. Two-dimensional Monte Carlo simulation based on the secondary emission electron avalanche model has been performed to analyze the charging progress. During the delay period, positive charge accumulates on the surface of the insulator near the cathode, which further accelerates the charging.
\end{abstract}

\section{INTRODUCTION}

Crair HARGING along the surface of an insulating spacer in vacuum is believed to trigger flashover, as reviewed in $[1,2]$. Understanding of the temporal behavior of the charging, however, seems to be inadequate. For example, based on the secondary electron emission avalanche (SEEA) mechanism proposed by Boersch et al. [3], Anderson [4] explained the voltage-time $(V-t)$ characteristic of a bridged vacuum gap exposed to a short voltage pulse. A space charge effect due to the ionization of desorbed gases in vacuum also was taken into account. In the theory, however, he assumed that the charging took place immediately after the application of the voltage, thus neglecting the time necessary for surface charging.

Recently we developed a probe method that enables us to make real-time observation of the surface charging in vacuum [5]. The probe measures the change in electric field at the cathode close to the triple junction where the insulator, cathode and vacuum meet, without disturbing the electric field in the gap or the charge distribution on the insulator. We have used this method for the investigation of the temporal behavior of charging on a cylindrical insulator exposed to a dc voltage step or a trapezoidal voltage. We have found that charging takes a substantial period of time after the voltage application.

\section{EXPERIMENTAL}

The details of the experimental setup and the procedure have been described in a previous paper [5], so we only explain the charge measuring technique briefly. The probe is a ring-shaped part isolated from the grounded plane cathode and is located coaxial with a cylindrical specimen as shown in Figure 1. The probe was grounded through a charge-measuring capacitor and the probe signal was converted into electric field strength $E_{T J}$, which is the sum of the geometrical field component $E_{g}=V_{a} / d$, where $V_{a}$ is the applied voltage, and $d$ the electrode separation, and the surface charge component $E_{s}$, where $E_{T J}=E_{g}+E_{s}$. The bandwidth of this measuring system is from dc to $10 \mathrm{MHz}$.

Test specimens were made of PMMA or alumina ceramic $\left(92 \% \mathrm{Al}_{2} \mathrm{O}_{3}\right)$ in the shape of a right cylinder with diameter $54 \mathrm{~mm}$ and height $10 \mathrm{~mm}$. The surface of each PMMA specimen was machined and had an average roughness of $r_{a}=0.71 \mu \mathrm{m}$, and maximum roughness $r_{\text {max }}=10.3 \mu \mathrm{m}$. The $\mathrm{Al}_{2} \mathrm{O}_{3}$ specimens were unglazed and had an average roughness of $r_{a}=1.38 \mu \mathrm{m}, r_{\max }=11.0 \mu \mathrm{m}$. We also examined for comparison polished PMMA specimens with average roughness of $r_{a}=0.13 \mu \mathrm{m}$, $r_{\text {max }}=1.69 \mu \mathrm{m}$, and polished $\mathrm{Al}_{2} \mathrm{O}_{3}$ specimens with average roughness of $r_{a}=0.16 \mu \mathrm{m}, r_{\max }=3.34 \mu \mathrm{m}$. The relative permittivity $\varepsilon_{r}$ of PMMA changes from 3 to 4 depending on the frequency of the applied 
IEEE Transactions on Dielectrics and Electrical Insulation

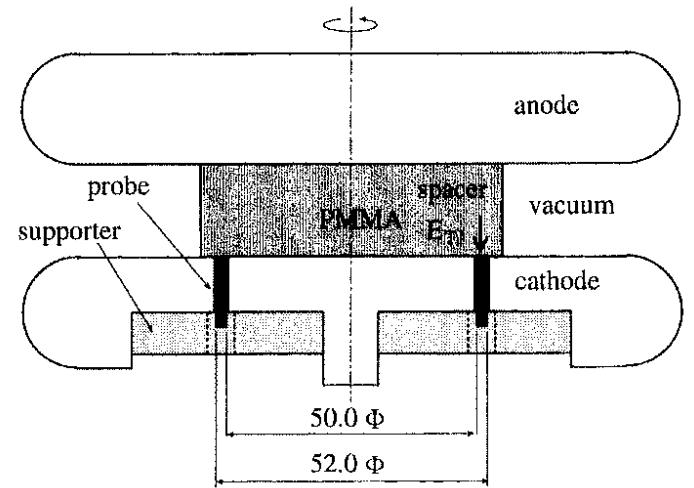

Figure 1. Arrangement of probe, insulator and electrodes.

voltage, while $\varepsilon_{r}$ of $\mathrm{Al}_{2} \mathrm{O}_{3}$ is $\sim 9$, independent of the frequency.

These specimens were subjected to a dc voltage step or a trapezoidal voltage. We generated the step voltage from a dc generator through a mechanical switch, and the trapezoidal voltage by substituting a $\mathrm{HV}$ semiconductor switch (Pulse Electric Co., HVS-36) for the mechanical switch. The voltage width ranged from $100 \mu$ s to $3 \mathrm{~ms}$. We used the step voltage with a delay $>3 \mathrm{~ms}$ before charging began.

\section{PROBE SIGNAL}

Figure 2 shows an example of the probe signal when a PMMA specimen is subjected to a dc voltage step. The field shows two rapid increases, first at the beginning of the voltage application $\left(E_{g}\right)$ at time $t=0$, and secondly due to the surface charging $\left(E_{s}\right)$ occurring at $t=340 \mathrm{~ms}$. Also, it can be seen that each component increases slowly after the rapid rise. We attribute the slow increase to the change in the permittivity of PMMA with frequency, since this does not happen when alumina specimens are tested. In this paper, we define the interval between the first and the second field rise as the 'surface charging delay time'.

The surface charge component keeps its value almost constant even after the applied field diminishes [3]. The remanent field is due to the positive charge remaining on the solid dielectric surface. We neutralized the residual charge by a silent discharge caused by introducing a small amount of air into the vacuum vessel after voltage removal [5].

\section{ELECTRIC FIELD STRENGTH NEAR THE TRIPLE JUNCTION}

We have reported the influence of applied voltage on the surface charging in previous papers $[5,6]$. When a specimen was subjected to a ramped dc voltage, the surface charge component $E_{s}$ increased almost linearly with the applied voltage [5], and $E_{s}$ depended strongly on the specimen surface roughness [6]. Figure 3 shows the electric field $E_{T} J$ as a function of the applied voltage $V_{a}$ for two $\mathrm{Al}_{2} \mathrm{O}_{3}$ specimens with smooth and rough surfaces, $r_{a}=0.16$ and $1.38 \mu \mathrm{m}$ respectively, when the stepwise dc voltage is applied. In each case, the average of $E_{s}$ increases almost linearly with $V_{a}$, as is the case when the ramped dc voltage is applied. It is surprising that the smoother surface acquires a charge three to four times higher than the rough surface.
Vol. 7 No. 6, December 2000

813
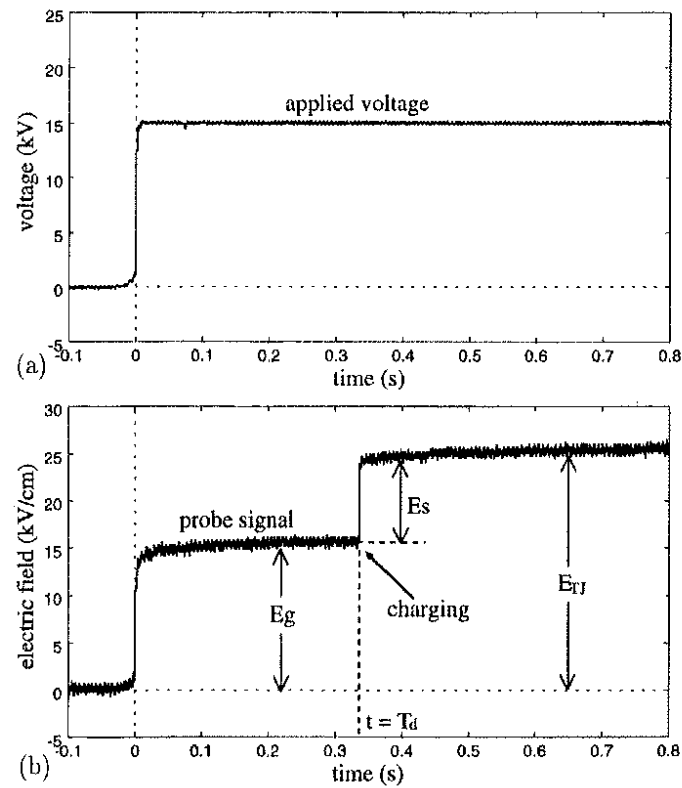

Figure 2. Wave forms of applied voltage and probe signal (PMMA, normal contact. $r_{a}=0.71 \mu \mathrm{m},+15 \mathrm{kV}$ stepwise voltage), (a) voltage, (b) probe signal.

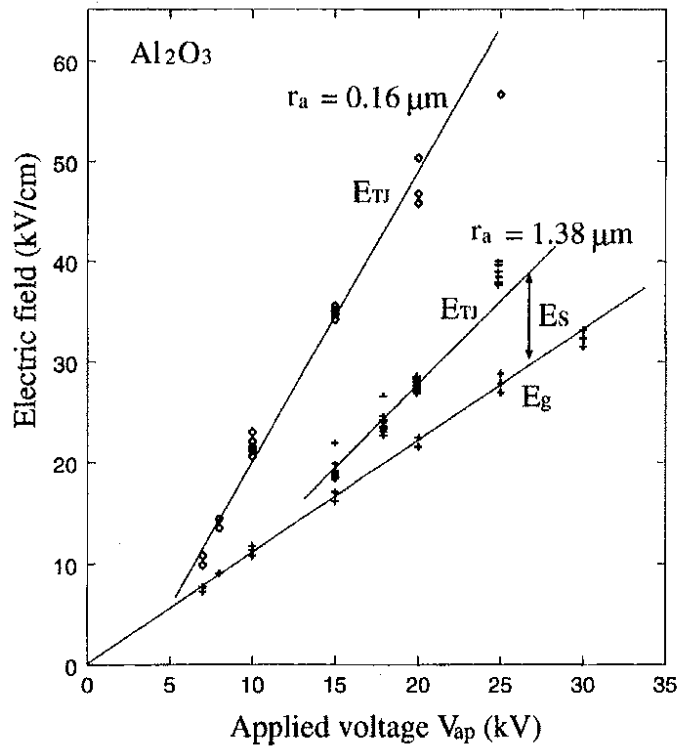

Figure 3. Electric field as a function of applied voltage for different surface roughness $\left(\mathrm{Al}_{2} \mathrm{O}_{3}\right.$, normal contact).

\section{DELAY TIME CHARACTERISTICS}

\subsection{FUNDAMENTAL RESULTS}

A specimen was repeatedly subjected to voltages at intervals of $\sim 10 \mathrm{~min}$, which roughly corresponded to the time necessary for removing the surface charge by the silent discharge and for the subsequent evacuation of gas from the test vessel. We have found that the 
delay time depends on this interval time. When the time interval is large, $24 \mathrm{~h}$ for example, the delay time exceeds $\sim 30 \mathrm{~s}$, much longer than in the case when the interval is $10 \mathrm{~min}$. Thus, it is important to keep the interval as constant as possible during the test.
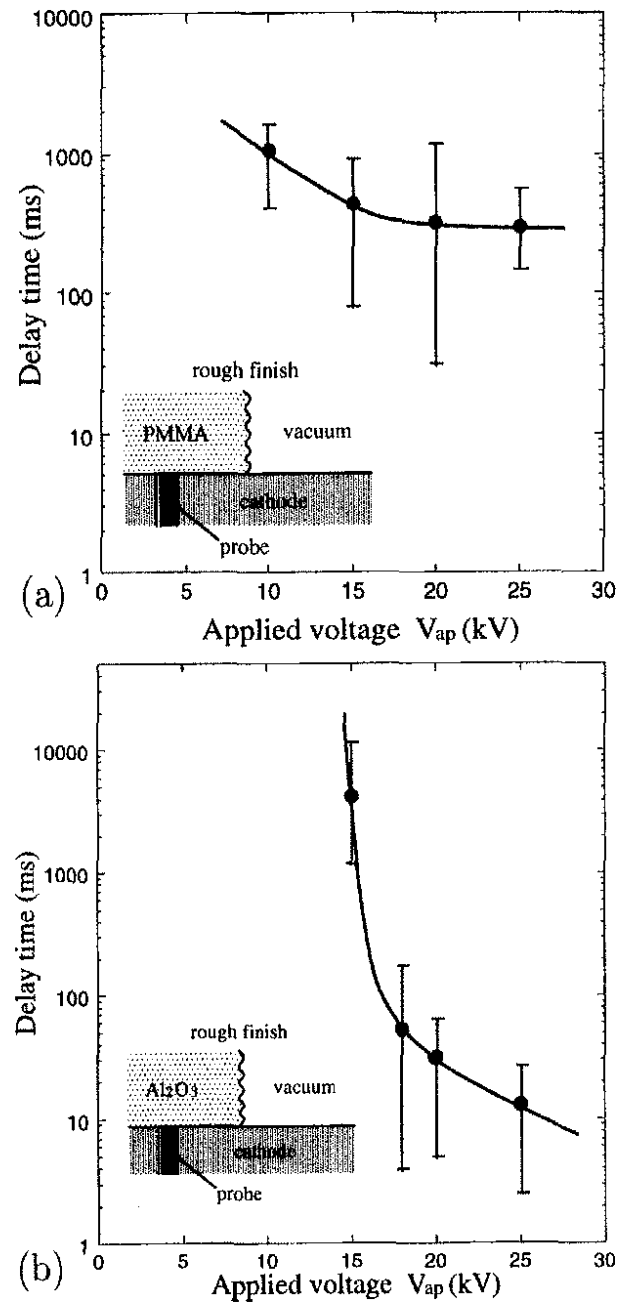

Figure 4. Delay characteristics for insulators with a comparatively rough surface (normal contact), (a) PMMA, $r_{a}=0.71 \mu \mathrm{m}$, (b) $\mathrm{Al}_{2} \mathrm{O}_{3}$, $r_{a}=1.38 \mu \mathrm{m}$

Figures 4(a) and (b) show the average delay time $t_{a v}$ together with the observed scatter (maximum and minimum delay time obtained for 10 trials) as a function of $V_{a}$ for PMMA and $\mathrm{Al}_{2} \mathrm{O}_{3}$ specimens. The measured time shows a large scatter. In the case of PMMA (Figure 4(a)), $t_{a v}$ decreases from $1.1 \mathrm{~s}$ at $V_{a}=10 \mathrm{kV}$ to $0.32 \mathrm{~s}$ at $V_{a}=20 \mathrm{kV}$, and becomes almost constant at $\sim 0.30 \mathrm{~s}$ on the average.

The delay time characteristic of $\mathrm{Al}_{2} \mathrm{O}_{3}$ specimens is similar, but decreases faster than that of the PMMA specimens as seen in Figure 4(b). The delay time is influenced by various experimental conditions as described in the following Sections.

\subsection{CONTACT CONDITION}

The experiments described above were carried out with the electrode system in which each end of the specimen simply contacted the corresponding electrode surface (called 'normal contact' in this paper). The experiment described in this Section was performed with an artificial small gap at the cathode. We inserted a film made of polyethylene terephthalate (PET), having diameter of $53 \mathrm{~mm}$ and thickness of $0.1 \mathrm{~mm}$ between the PMMA specimen and the cathode as illustrated in Figure 5. Because the diameter of the PET film is $1 \mathrm{~mm}$, smaller than that of the PMMA specimen, a uniform small gap is formed at the periphery of the cathode side of the specimen, thereby enhancing the electric field by a factor of approximately $\varepsilon_{r}=3$ to 4 depending on the frequency.

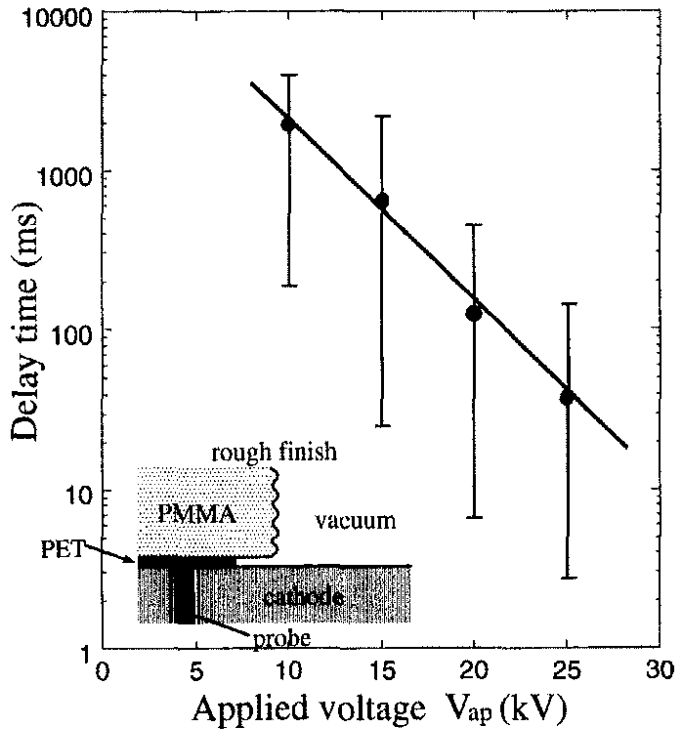

Figure 5. Delay characteristic with an artificial gap (PMMA, $\left.r_{a}=0.71 \mu \mathrm{m}\right)$

The delay time in Figure 5 decreases almost linearly on a semilogarithmic scale, showing that charging progresses faster for higher voltages when compared with the normal contact (Figure 4(a)). This is especially so for $V_{a}>20 \mathrm{kV}$. We believe that the field emission of electrons increases due to the enhanced electric field, thus accelerating the charging.

\subsection{INFLUENCE OF INSULATOR SURFACE ROUGHNESS}

The experimental results mentioned above are concerned with insulators having a comparatively rough surface $\left(r_{a}=0.71 \mu \mathrm{m}\right.$ for PMMA and $r_{a}=1.38 \mu \mathrm{m}$ for $\mathrm{Al}_{2} \mathrm{O}_{3}$ ). The delay time with a polished PMMA specimen is shown in Figure 6(a) and for a polished $\mathrm{Al}_{2} \mathrm{O}_{3}$ specimen in Figure $6(b)$. These results demonstrate that the delay time of polished specimens is much shorter than that of the unpolished ones. The average delay times in these Figures show complicated variations with $V_{a}$. However, the minimum delay times decrease linearly with $V_{a}$ on a semi-logarithmic scale. 

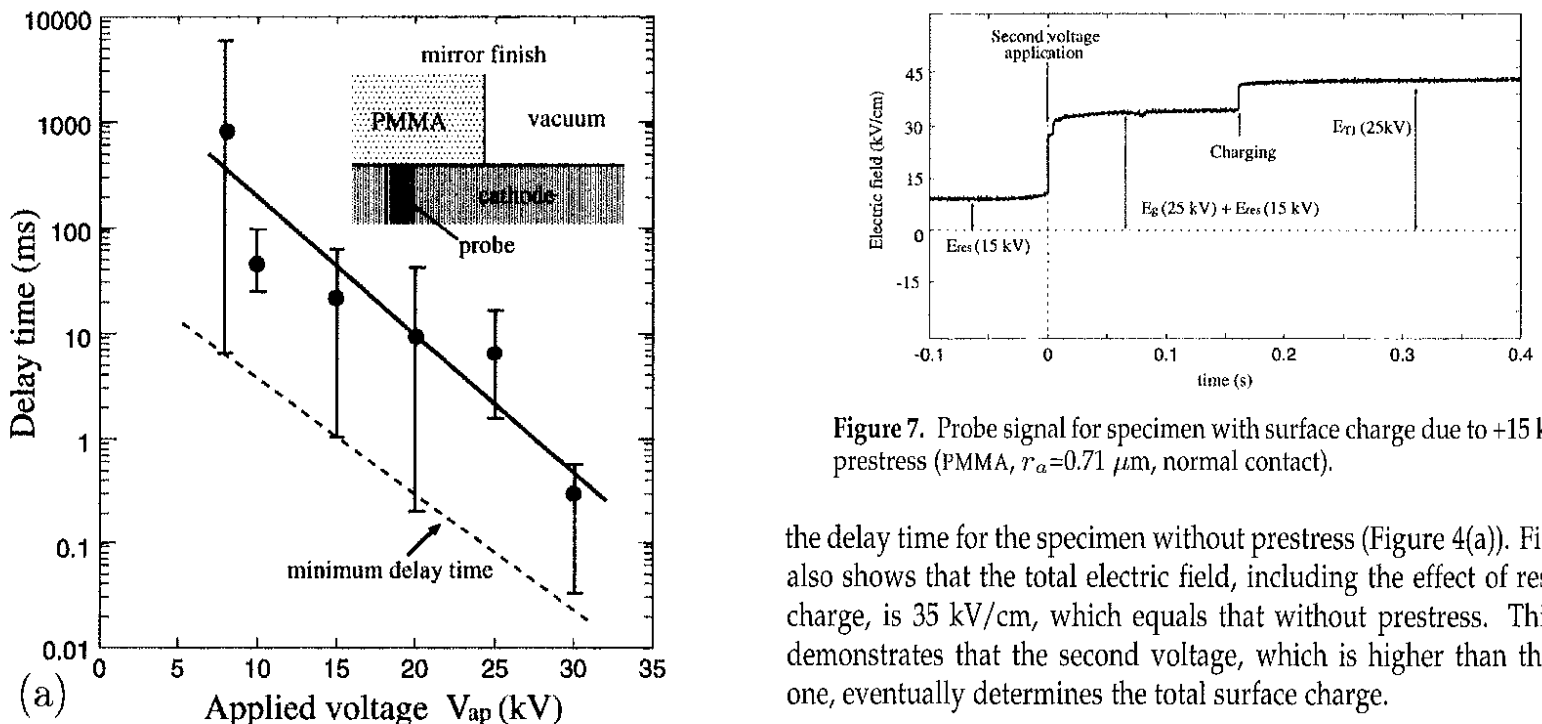

Figure 7. Probe signal for specimen with surface charge due to $+15 \mathrm{kV}$ prestress (PMMA, $r_{a}=0.71 \mu \mathrm{m}$, normal contact).

the delay time for the specimen without prestress (Figure 4(a)). Figure 7 also shows that the total electric field, including the effect of residual charge, is $35 \mathrm{kV} / \mathrm{cm}$, which equals that without prestress. This fact demonstrates that the second voltage, which is higher than the first one, eventually determines the total surface charge.

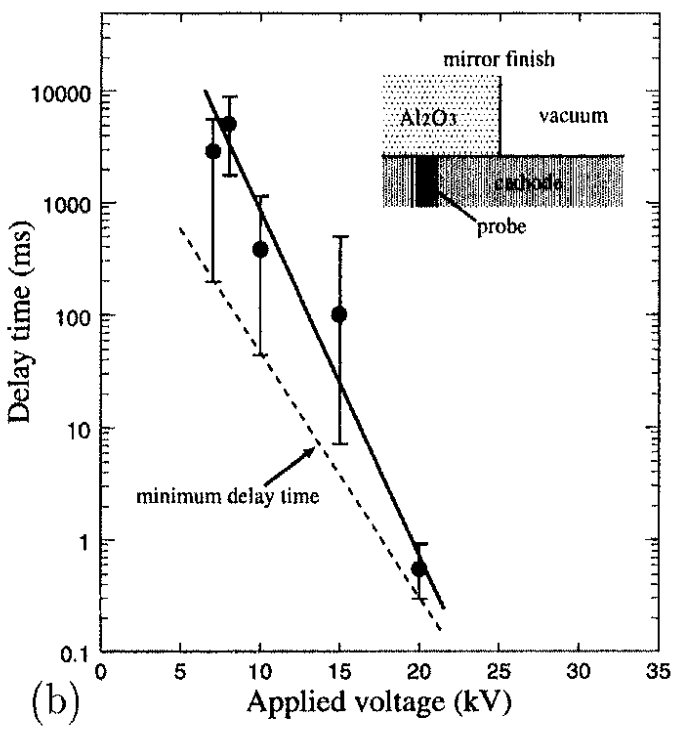

Figure 6. Delay characteristics for insulators with comparatively smooth surfaces (normal contact), (a) PMMA $\left(r_{a}=0.22 \mu \mathrm{m}\right.$ ), (b) $\mathrm{Al}_{2} \mathrm{O}_{3}$ $\left(r_{a}=0.16 \mu \mathrm{m}\right)$

\subsection{INFLUENCE OF PRESTRESS}

We applied the dc voltage step twice to an insulator without applying the silent discharge procedure after the first voltage application. The interval between the two applications was $\sim 3$ min, thus preserving charge due to the first voltage. Figure 7 shows an example of the probe signal when the first voltage is $15 \mathrm{kV}$ and the second one is $25 \mathrm{kV}$ at $t=0 . E_{r}=10 \mathrm{kV} / \mathrm{cm}$, denotes the electric field caused by the residual charge due to the first voltage. Since this is same as $E_{s}$ in Figure 2, we have confirmed that the surface charge due to the first voltage is preserved. According to a calculation based on the SEEA mechanism [7], the surface acquires positive charge approximately $20 \mu \mathrm{C} / \mathrm{m}^{2}$ at $15 \mathrm{kV}$.

The charging due to the second voltage occurs at $60 \mathrm{~ms}$, on the average, measured from the second voltage rise. This is almost one fifth of

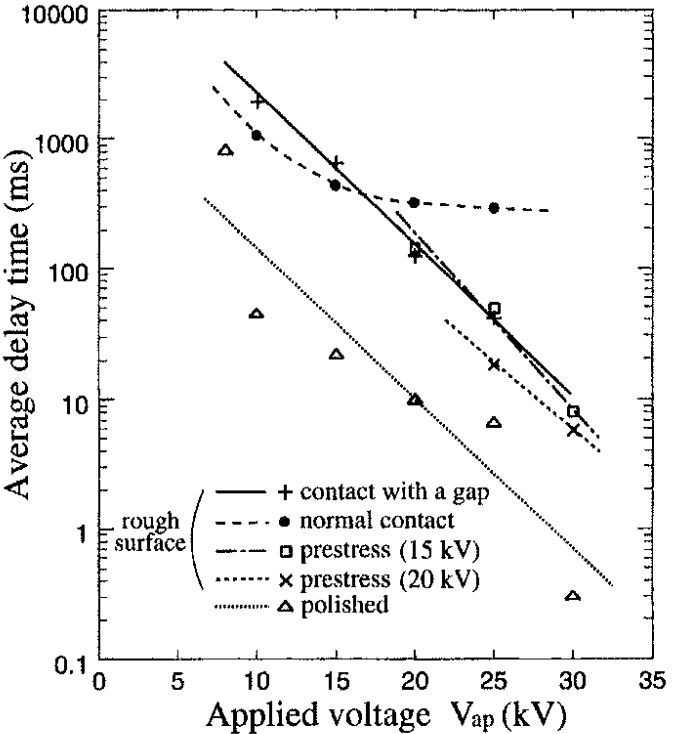

Figure 8. Delay time for PMMA specimens under various experimental conditions.

The average delay time characteristics of PMMA samples for various conditions are compared in Figure 8. Note that the delay time for the prestress at $15 \mathrm{kV}$ almost agrees with that of the experiment with the artificial small gap, and that the delay time becomes even shorter when the prestress voltage is increased to $20 \mathrm{kV}$.

\section{FLASHOVER}

Applying the voltage occasionally leads to flashover along the surface of the sample when the voltage is higher than a certain value. Figure 9 shows probe signals when an alumina specimen is subjected to a trapezoidal voltage. Figures $9($ a) and (b) show the applied voltage and probe signal when the charging occurs at $t=2.6 \mathrm{~ms}$ without developing as far as flashover, while Figure 9(c) shows the probe signal when flashover takes place. 


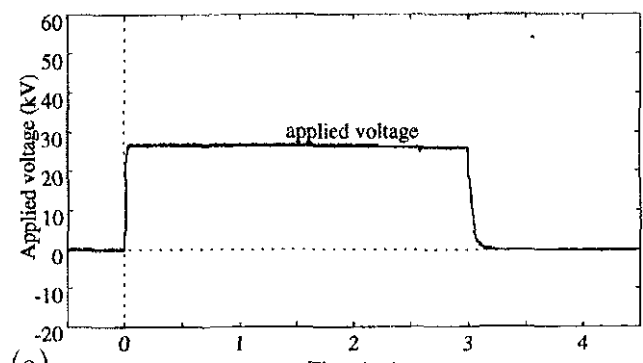

(a)
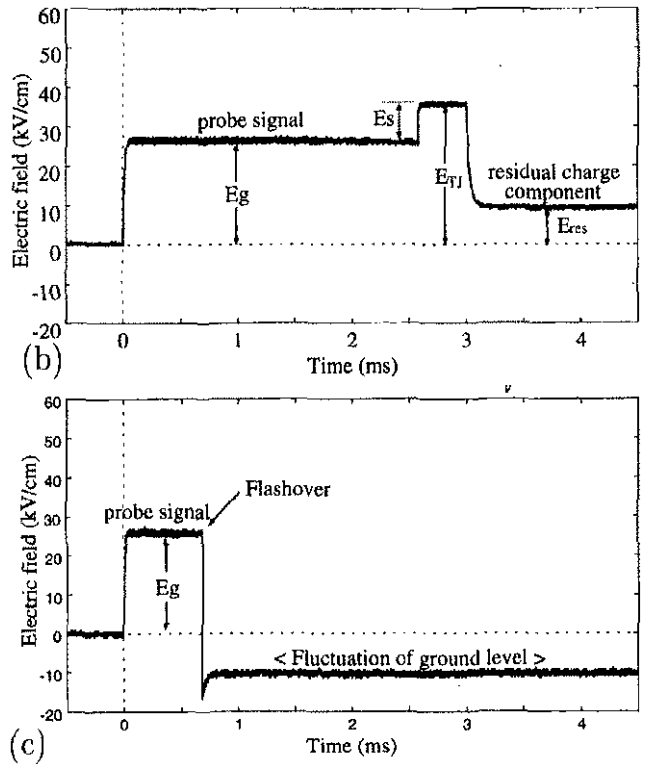

Figure 9. (a) Applied voltage and (b) probe signals without flashover and (c) with flashover $\left(\mathrm{Al}_{2} \mathrm{O}_{3}\right.$, normal contact).

In the case of ramped dc voltage application, we can identify flashover by a sudden voltage drop and a bright flash after the surface charge field component $E_{s}$ has increased [5]. It can be seen in Figure 9(c) that the interval between the rise in $E_{s}$ and the flashover is too short to measure with our probe. We infer that the flashover occurs immediately after the charging in the case of trapezoidal or stepwise dc voltage application.

\section{ANALYSIS ON PROGRESS OF CHARGING}

In our previous paper [8], we have studied numerically the delay time of charging by applying a two-dimensional Monte Carlo simulation method based on the SEEA mechanism. Figure 10 shows an example of the calculated probe signal for a PMMA specimen, where the space charge component $E_{s}$ increases abruptly at a time $T_{d}=340 \mathrm{~ms}$. The simulated signal basically agrees with the experimental one depicted in Figure 2. In the following, we explain the calculated waveform through charge distribution along the specimen.

Figure 11 shows the calculated charge distributions along the insulator at three different time ranges. The simulation gives electric field distributions (normalized to the average field strength $E_{a v}$ ) on

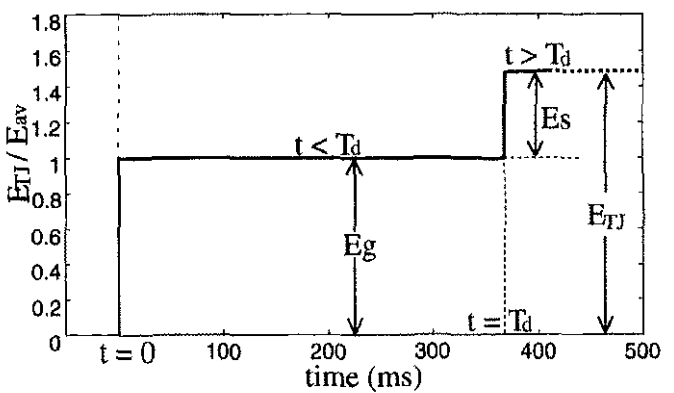

Figure 10. Calculated probe signal (PMMA).

the cathode near the triple junction for the three charge distributions as represented in Figure 12.

Figure 11(a) is the distribution at a time just before the beginning of the rise in $E_{s}\left(t<T_{d}\right)$. The Figure shows that only a narrow band of the insulator, very close to the cathode, acquires a small amount of positive charge. It can be seen from Figure $12\left(t<T_{d}\right)$ that the electric field on the cathode is too weak to be detected by the probe.

At $t \simeq T_{d}$, the charge amount becomes so large as to enhance the junction field considerably as shown in Figures $11(\mathrm{~b})$ and $12\left(t \simeq T_{d}\right)$. We have calculated that electron emission from the cathode junction increases markedly and thus accelerates the charging. At this moment, the electric field begins to increase abruptly. The charge distribution and consequent electric field just after the time $T_{d}$ are shown in Figures $11(c)$ and $12\left(t>T_{d}\right)$, respectively. We believe that the positive feedback phenomenon mentioned above makes the charging propagate rapidly toward the anode. It has been reported that the charging progresses at a speed of $2 \mathrm{~ns} / \mathrm{mm}$ [9].

The analysis indicates that the delay time corresponds to the time necessary to accumulate positive charge large enough to start the positive feedback phenomenon. Although the field emission of electrons from the cathode strongly depends on the electric field, the current due to the geometric electric field is very low. Therefore, we consider that it needs an extended time before the feedback becomes effective. The simulation gives a delay time $>1 \mathrm{~s}$ as observed in the experiment. Therefore, the SEEA mechanism accounts for the surface charging process for both pulsed voltage excitation and dc excitation in vacuum.

When the positive feedback occurs, the electric field at the triple junction immediately increases by $\approx 10 E_{a v}$ as seen in Figure 12 . This field increase may bring considerable field emission of electrons. Thus, we believe that feedback is essential for the progress of surface charging and eventual flashover.

\section{CONCLUSION}

THE present study has revealed that there is considerable delay between voltage application and charging in vacuum. The delay time ranges from $10^{-5} \mathrm{~s}$ to $10 \mathrm{~s}$. The delay shows considerable dispersion. However, it decreases almost linearly with the voltage on a semilogarithmic scale. The delay is influenced by the various experimental conditions such as the material used, the surface roughness, the state of the contact at the insulator-cathode interface and the prestress. Among all the experimental conditions, polishing the insulator surface has the 


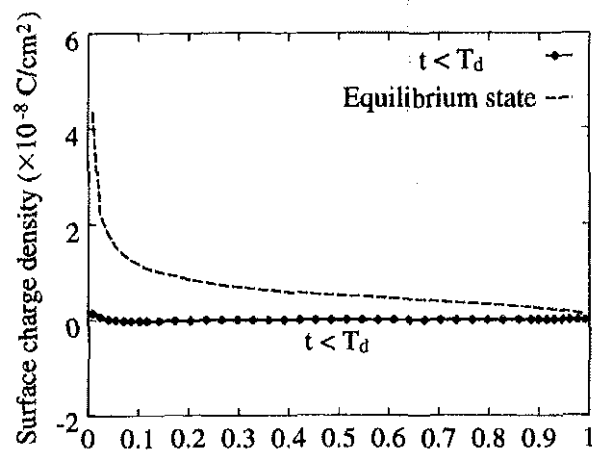

(a) Distance from cathode along the surface $(\mathrm{cm})$

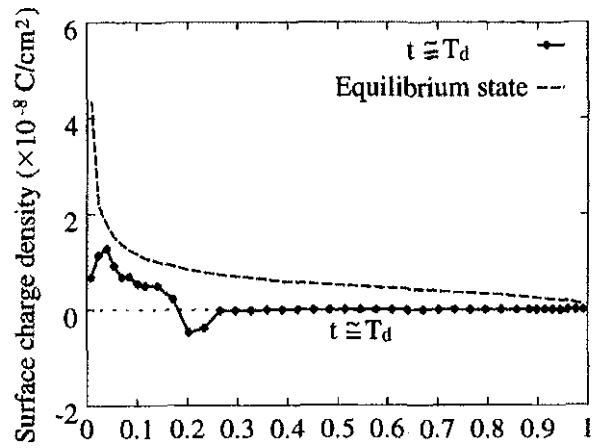

(b) Distance from cathode along the surface $(\mathrm{cm})$

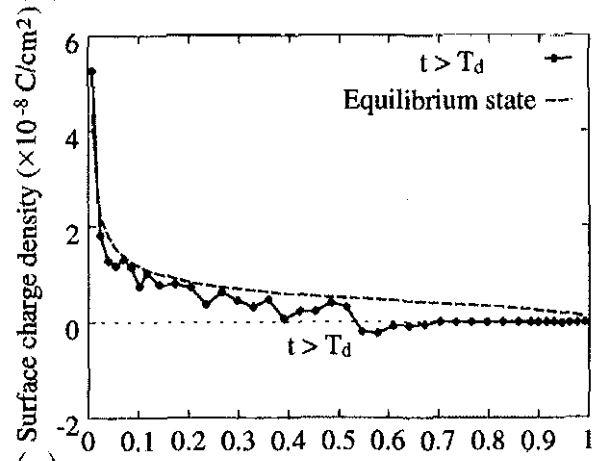

(c) Distance from cathode along the surface $(\mathrm{cm})$

Figure 11. Calculated surface charge distributions for (a) $t<T_{d}$, (b) $t \simeq T_{d}$ and (c) $t>T_{d l}$ (PMMA).

largest effect on promoting the charging of an insulator in vacuum. The analysis of the surface charge and the electric field distributions shows that the delay time corresponds to the time necessary to accumulate positive charge large enough to start a positive feedback effect that increases the junction field and, therefore, the field emission of electrons. The feedback phenomenon is essential for the progress of the charging

The cause of the surface-polishing effect on charging and the origin of the large dispersion of the delay time are not clear at this moment, and need to be studied.

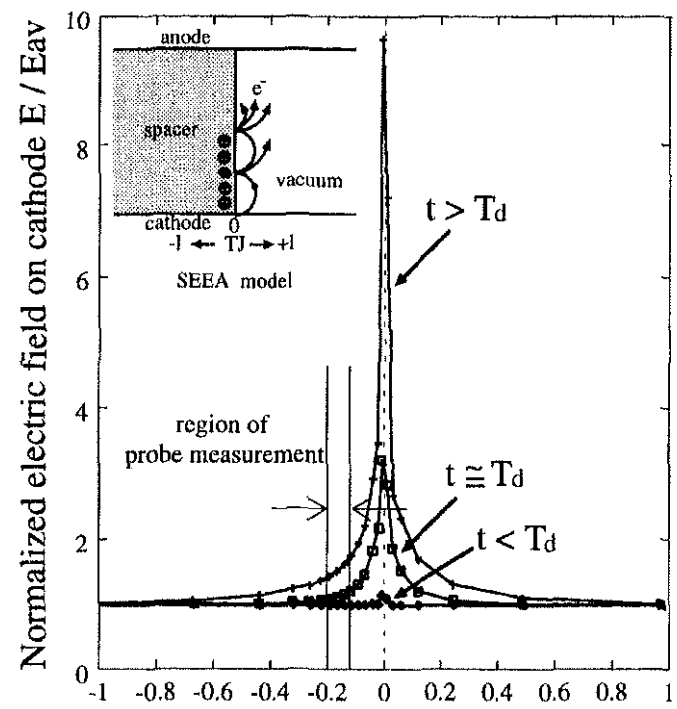

Distance from cathode $\mathrm{TJ}(\mathrm{cm})$

Figure 12. Calculated electric field distributions for the three time ranges on the cathode near the triple junction (PMMA).

\section{ACKNOWLEDGMENT}

This study is supported by a grant-in-aid from the Japanese Ministry of Education.

\section{REFERENCES}

[1] R. A. Anderson, "Surface flashover: Three decades of controversy", Proceedings of 14th Int. Conf. on Discharges and Electrical Insulation in Vacuum, Santa Fe 1990, pp. 311-317, 1990.

[2] H. C. Miller, "Flashover of insulators in vacuum", IEEE Trans. Electr, Insul., Vol, 28 pp. 512-527, 1993

[3] H, Boersch, H. Harmish and W. Ehrlich, "Surface discharge across insulators in vacuum", Z. Angew Phys, Vol. 15, pp. 518-525, 1963.

[4] R. A. Anderson and J. P. Brainard, "Mechanism of pulsed surface flashover in vacuum", I. Appl. Phys., Vol. 51, pp. 1414-1421, 1980

[5] O. Yamamoto, T. Takuma and T. Tanabe, "Real-time observation of surface charging on a cylindrical insulator in vacuum", IEEE Trans. Dielectrics EL, Vol. 5, pp. 961-965 1998

[6] O. Yamamoto, T, Takuma, S. Hamada, Y. Kakehashi, S. Ikoma, K. Nishimura and $\mathrm{T}$. Shioiri, "Influence of surface roughness on charging of an insulating spacer in vacuum", JIEE Meeting on Electrical Discharges, ED-98-206 (in Japanese), pp. 4349,1998

[7] O. Yamamoto, T. Hara, I. Nakanishi and M. Hayashi, "Monte Carlo simulation of surface charge on angled insulators in vacuum", IEEE Trans. Electr. Insul., Vol. 28, pp. 706-712, 1993 .

[8] O. Yamamoto, T. Hara, H. Matsuura and M. Hayashi, "Temporal Behavior of Surface Charge Accumulation in Bridged Vacuum Gaps", IEEE Trans. Dielectrics EI, Vol. 2 , pp. 292-298, 1995.

[9] J. E. Thompson, J. Lin, K. Mikkelson and M. Kristiansen, "Investigation of fast insulator surface flashover in vacuum", IEEE Trans. Power App. Syst., Vol. 8, pp. $191-197,1980$

Manuscript was received on 11 February 2000, in final form 12 July 2000. 